\title{
À PROPOS DES NOMINA ACTIONIS DES VERBES DE PERCEPTION VISUELLE POLONAIS ET FRANÇAIS
}

\author{
Dorota ŚLIWA \\ Université catholique Jean-Paul II de Lublin
}

\begin{abstract}
The nomina actionis of the selected verbs of visual perception are studied here in their discursive function in a well-defined context, namely that of the meetings between Jesus and Sister Faustina Kowalska, described in her "Diary". This communication situation will make it possible to locate the Polish and French derivational models for the nouns spojrzenie / widzenie - regard / vision, to propose a coherent semantic description of these nouns which takes into account its different dimensions and to indicate the importance of the collocative verbs for the study of the meaning of nomina actionis. The article also aims to be a contribution of the linguistic analysis of the parallel bilingual corpus for the contrastive approach of Polish and French derived nouns.
\end{abstract}

\section{INTRODUCTION}

Dans son étude sur la sémantique des noms d'action en français, Richard Huyghe constate que ces noms sont une « pierre angulaire du lexique nominal » (2014 : 181). Ils sont généralement étudiés du point de vue du modèle dérivationnel ou du point de vue syntaxique (nominalisation des phrases). Il existe cependant des approches cognitives et pragmatiques de la formation des nomina actionis, initiées déjà dans les années soixante-dix du XX $\mathrm{XX}^{\mathrm{e}}$ siècle par Maria Honowska (1979), continuées par Krystyna Waszakowa (2015). Ces approches rejoignent une optique dénominative, incluant la structure ontologique et l'activité cognitive du sujet parlant, que nous avons proposée en 2000.

L'étude des nomina actionis des verbes de perception visuelle se veut un réexamen du sens de ces noms et de leur fonctionnement en discours. Pour ce faire, nous analyserons en détail les exemples relevés du corpus bilingue parallèle : le 
texte original polonais de Dzienniczek ${ }^{1}$ (1934-1938) de Faustyna Kowalska, et sa deuxième traduction en français, Petit Journal (2017) ${ }^{2}$. En un premier temps, nous rappellerons les informations élémentaires concernant la perception visuelle afin de donner un cadre ontologique pour les prédications sous-jacentes à la formation des nomina actionis et pour l'activité perceptive de Jésus et de Sœur Faustine (désormais : SF) décrite dans le discours mystique. En un deuxième temps, nous décrirons les emplois des noms d'action dans diverses constructions syntaxiques avec des verbes collocatifs qui réalisent les différentes prédications sur l'activité perceptive des personnes concernées.

\section{LA PERCEPTION VISUELLE ET SA DÉNOMINATION PAR DES NOMINA ACTIONIS}

Les nomina actionis dénommant la perception visuelle nécessitent un cadre ontologique complet qu'est la personne et son organe qui réalise le sens de la vision (vue). Ce cadre est actualisé par les personnes décrites dans le discours spécialisé et sert de référence aux structures prédicatives sous-jacentes aux exemples analysés.

\subsection{L'ONTOLOGIE DE LA PERCEPTION VISUELLE - INFORMATIONS ÉLÉMENTAIRES}

La perception visuelle a pour prédication de base la structure prédicative suivante : une personne voit quelque chose avec les yeux. Cette prédication implique une personne et son organe - l'œil - dont la fonction principale est celle de voir (la vision). La perception visuelle est donc l'action directe de l'œil, défini comme « organe de la vision $»^{3}$. Les spécialistes ${ }^{4}$ remarquent aussi que les " yeux transmettent des flux continus d'images au cerveau par l'intermédiaire des signaux électriques » et qu'ils " reçoivent l'information à partir des rayons lumineux ». Sans entrer dans la description détaillée, soulignons ici le lien entre l'action des signaux électriques (lumière) sur les yeux, la formation des images et leur transmission au cerveau ; il s'agit donc du lien entre la perception visuelle

\footnotetext{
${ }^{1}$ Accesible en ligne : www.faustyna.pl/zmbm/dzienniczek-sw-siostry-faustyny (dernière consultation : le 03.01.2018).

${ }^{2}$ Sœur Faustine Kowalska, Petit Journal, texte accessible en ligne : https://www.faustyna.pl/ $\mathrm{zmbm} / \mathrm{fr} /$ texte-integral-du-petit-journal (dernière consultation : le 03.01.2018).

${ }^{3} \mathrm{http} / / /$ dictionnaire.academie-medecine.fr/?q=oeil (dernière consultation : le 10.01.2018).

${ }^{4} \mathrm{http} / / /$ dictionnaire.doctissimo.fr/definition-oeil.htm (dernière consultation : le 10.01.2018).
} 
et l'activité du cerveau. Ce lien est essentiel pour le sens de la vision, définie par les spécialistes ${ }^{5}$ comme « un processus très complexe qui nécessite la participation de nombreux éléments des yeux et du cerveau ». Dans l'ensemble, il s'agit de la capacité de capter des rayons lumineux porteurs des informations (images) et de les analyser.

L'organe de la vision est dénommé par les termes: oko / oczy - cil / yeux ; le processus par: wzrok-vision, vue. Sur le plan ontologique, ce sont les méronymes désignant l'action d'un organe de la personne désignée par l'holonyme osoba - personne. Le rappel de ces informations fondamentales est ici nécessaire pour expliquer par la suite les glissements sémantiques opérés sur cette prédication élémentaire.

\subsection{LA FORMATION DES NOMINA ACTIONIS EN LATIN, EN POLONAIS ET EN FRANÇAIS}

La prédication de base ci-dessus sert de paraphrase dérivationnelle. Suivant les modèles dérivationnels communément admis dans toutes les approches théoriques (structuralistes et générativistes) de la dérivation suffixale, les noms d'action sont formés à partir de la base verbale avec les suffixes qui ont le sens 'action de V'. Dans la formation des nomina actionis des verbes de perception visuelle, ce sont : le suffixe latin -tiō (et sa forme alternative -siō), emprunté par le polonais (-acja) et par le français (-ation) ; ensuite le suffixe polonais -anie / -enie ; le suffixe français -ment, etc.

Ainsi le mot latin visio est formé selon le modèle vīdeō $+-t i \bar{o}$. Il est emprunté en polonais (wizja) et en français (vision). D'autres emprunts au latin : en polonais : obserwować-obserwacja; kontemplować-kontemplacja; en français : observer-observation; contempler-contemplation.

En polonais, les nomina actionis sont formés à partir des verbes perfectifs avec les préfixes qui précisent le sens spatial : spo- selon la direction du haut vers le bas (spojrzeć - spojrzenie) et we- selon la perception en profondeur (wejrzeć - wejrzenie). Le verbe imperfectif widzieć et son nom d'action widzenie désigne la capacité de voir ou l'action dans sa durée. En français, les nomina actionis des verbes de perception visuelle sont soit empruntés au latin, comme vision qui est une forme supplétive du verbe voir, soit formés par conversion du participe passé $v u$ en nom (la) vue désignant la capacité de voir, soit créés par la suffixation zéro comme regard à partir du verbe regarder.

${ }^{5} \mathrm{http}: / /$ dictionnaire.doctissimo.fr/definition-vision.htm (dernière consultation : le 10.01.2018). 
Les nomina actionis sont des noms prédicatifs, nécessitant pour la description sémantique la structure prédicative avec des lexèmes instanciant le rôle d'agent, d'objet / patient, d'instrument, etc., comme par exemple pour le prédicat regarder : quelqu'un regarder quelqu'un / quelque chose avec les yeux-regard de quelqu'un sur quelqu'un / quelque chose. Au niveau de la paraphrase dérivationnelle, nous notons une fréquente métonymie actancielle pour le sens structurel du suffixe : 'action de V' $\rightarrow$ 'résultat de l'action de V', notamment pour le nom widzenie - vision.

\subsection{LES NOMINA ACTIONIS EN DISCOURS MYSTIQUE CATHOLIQUE}

Suivant l'approche dénominative de la dérivation, les noms d'action se forment en discours et deviennent ensuite un signe linguistique avec son pouvoir référentiel indépendant du sens structural donné par la paraphrase dérivationnelle. C'est une nouvelle unité dénominative en discours, en l'occurrence en discours mystique catholique.

Il s'agit d'un type de discours spécialisé, censé décrire une réalité de manière précise, comme le font pour d'autres réalités des textes techniques, juridiques, scientifiques, etc. La spécificité du discours mystique catholique tient au fait qu'il décrit une réalité spirituelle par ceux qui la voient et que celle-ci est ensuite authentifiée par les spécialistes (théologiens). Le discours d'un mystique catholique n'est pas restreint au texte littéraire (supposé décrire une réalité suivant l'intuition d'un littéraire qui s'exprime dans un style littéraire), même s'il se caractérise par un style d'écriture (figures rhétoriques, lexique) qui exprime avec beauté la réalité décrite. Nous pouvons définir sommairement le discours mystique comme discours spécialisé du domaine de la théologie dans ce sens qu'il implique la connaissance de la Bible et de la pratique religieuse et qu'il est compréhensible pour la communauté des sujets parlants remplissant ces conditions.

Le chef d'œuvre de la littérature mystique, Dzienniczek (Petit Journal) de Sœur Faustine a été traduit en plusieurs langues (en français il y a deux versions de la traduction). L'étude des verbes de perception visuelle, très fréquemment employés par la mystique, est intéressante de divers points de vue. Tout d'abord, les nomina actionis de ces verbes sont employés dans un contexte bien défini, celui de ses rencontres mystiques avec Jésus (Sœur Faustine l'explique elle-même dans ses définitions de widzenie qui seront présentées sous 2.3.), et réfèrent donc à la réalité déterminée par les personnes, par le temps et par le lieu (dont les indices sont donnés dans le texte). Et finalement, l'étude du texte bilingue parallèle aboutit à la description des usages de ces noms en polonais (texte original) et en français (texte traduit) avec leurs caractéristiques morphosyntaxiques propres à chaque langue. 


\section{LES SENS DISCURSIFS DES NOMINA ACTIONIS}

Le sens discursif des nomina actionis est précisé par le verbe collocatif avec pour base le nom d'action, d'où l'importance des collocations comme prédication sur un aspect du concept (cf. Śliwa, 2002). Suivant l'approche dénominative de la dérivation, les nomina actionis spojrzenie - regard, widzenie - vision sont des prédications sur l'activité perceptive des deux personnes lors d'une rencontre mystique. Par conséquent, ils ont des structures prédicatives liées au contexte et modifiées en fonction de la prédication : sur les personnes, sur la capacité visuelle et sur le résultat de l'action perceptive.

\subsection{Prédications sur L'aCtivité perceptive d'une Personne : SPOJRZENIE / WZROK ET REGARD}

Les prédications sur l'activité perceptive d'une personne se caractérisent par la structure prédicative dans laquelle le lexème désignant Jésus ou Sœur Faustine se trouve en position de sujet d'un verbe collocatif (V) et le nom d'action est en position d'instrument ou d'objet.

Les classes sémantiques des verbes collocatifs de ces prédications sont les suivantes :

a) Les verbes de perception visuelle, notamment lorsque le sujet désigne Jésus. Ces verbes ont pour complément :

- les noms oko, wzrok - yeux : patrzeć okiem surowym na $(383)^{6}$; spogladać na coś wzrokiem surowym (1261) ; patrzeć na coś innym wzrokiem - regarder qqch avec d'autres yeux (104);

- ou, en français, le nom déverbal regard avec un collocatif (poser un regard sévère (383), ou sans nom d'organe ou de faculté de perception (regarder qqch avec sévérité (1261)).

b) Les verbes désignant un mouvement spatial :

- lorsque le sujet désigne Dieu : zwracać swe spojrzenie ku (383) - diriger son regard vers (383); zwracać swój wzrok ku (1339) - porter son regard sur (1339); odwracać swój wzrok od (1339) - détourner son regard de (1339) ;

\footnotetext{
${ }^{6}$ Les numéros des paragraphes du Petit Journal, les mêmes dans le texte original et dans le texte traduit.
} 
- lorsque le sujet désigne SF, notamment son âme, comme elle l'exprime elle-même : zatapiać wzrok swej duszy w-plonger le regard de son âme en (57), dans (681); zapuszczać wzrok swej duszy w-plonger le regard de son âme dans (1239); utkwić wzrok swej duszy w-fixer le regard de son âme sur $(1327,1333,1483)$.

c) Les verbes désignant l'activité intellectuelle accompagnée par la perception visuelle qui en devient l'instrument (rejoignant ainsi le lien entre les yeux et le cerveau). L'action désignée par le verbe polonais przeniknać est différemment traduite : celle de Jésus - pénétrer l'âme [de Sœur Faustine] (464) et celle de $\mathrm{SF}$ - scruter le monde (1632). L'activité intellectuelle est encore désignée par d'autres verbes collocatifs : obejmować coś wzrokiem - embrasser de son regard qqch (383) ; dawać komuś poznać spojrzeniem, że - faire comprendre par son regard que (1442) ; ...jednym spojrzeniem rozumiemy sie wzajemnie - ...nous nous comprenons d'un regard (201).

d) Les verbes désignant l'activité parlante accompagnée par la perception visuelle ont des structures syntaxiques spécifiques notamment en polonais, ce qui se traduit en français par des structures complexes coordonnées qui contiennent des prédications sur l'activité perceptive : Pan Jezus z łaskawym spojrzeniem rzekt [do siostry Faustyny]... - Jésus a regardé [Sceur Faustine] avec bienveillance et [lui] dit... (1782). Voir aussi (1346).

e) Notons encore les verbes désignant une autre activité accompagnée par la perception visuelle des personnes extérieures :

- lorsque le sujet désigne Jésus : chronić [Faustyne] przed wzrokiem [stworzeń, otoczenia, ludzi] - mettre à l'abri de [du regard des créatures (891), des regards de mon entourage (1054), des regards humains (1055)] ; protéger [Sœur Faustine] de [des regards humains] (1057);

- lorsque le sujet désigne SF : ukryć się przed wzrokiem ludzkim - se cacher au regard des hommes (306), se cacher aux yeux de son entourage (255).

Dans les prédications sur l'activité perceptive de Jésus ou de Sœur Faustine, le lexème polonais wzrok, dont le premier sens est celui de 'sens de la vision', a de fréquents emplois métonymiques. Le premier (a) est celui entre 'organe' $\rightarrow$ 'sens', où il concurrence avec oko; le deuxième (b), notamment pour 'sens' $\rightarrow$ 'action de' où il concurrence avec spojrzenie. Le lexème spojrzenie apparaît dans les contextes qui marquent l'activité de Jésus ou de Sœur Faustine (c, d). 


\subsection{SPOJRZENIE / WZROK ET REGARD : PRÉDICATIONS MÉTONYMIQUES SUR}

\section{LA VUE}

Les prédications métonymiques sur la vue, lorsque les lexèmes spojrzenie / wzrok et regard sont en position sujet d'un verbe collocatif, ont pour effet de mettre en relief l'action de voir, tout en reléguant la personne sur le fond de cette action (cf. 284). Le nom prédicatif spojrzenie - regard est suivi du complément du nom qui désigne l'agent qu'est Dieu : spojrzenie Boga - le regard de Dieu (890), spojrzenie Pana - le regard du Seigneur $(334,346)$; spojrzenie tego Mocarza - le regard de ce puissant Souverain (708) ; spojrzenie Jezusa - le regard de Jésus $(560,758)$. Seul le nom Bóg - Dieu est transformé en adjectif relatif : Boże spojrzenie - le regard de Dieu $(1025,1391)$; Boskie spojrzenie Jezusa - le regard divin de Jésus $(306,795)$.

\section{a) Attributs du regard}

Plusieurs prédicats adjectivaux désignent les propriétés du regard de Dieu. Ces adjectifs sont introduits par le verbe copule być - être : spojrzenie Pana jest stodkie - le regard du Seigneur est doux (560) qui est le plus souvent effacé pour former le syntagme nominal : wzrok święty - saint regard $(284,1339)$; wzrok surowy - regard sévère $(383,1261)$; miłosierne spojrzenie-regard miséricordieux (383) ; tagodne spojrzenie - doux regard (383); taskawe spojrzenie - regard bienveillant $(383,464,1782)$; glębokie spojrzenie - regard profond $(1442,1346$, 858) ; milosne spojrzenie Boga - regard amoureux de Dieu (890). Notons encore l'alternance de deux constructions : NAdj ou N peten - N plein de, $\mathrm{N}$ qui souligne l'intensité de la propriété désignée par Adj : wzrok peten głębi i przenikliwości - regard profond et pénétrant (434); milosne spojrzenie Twoje [Pana] - ton [du Seigneur] regard plein d'amour (334).

Le regard de SF est dénommé par wzrok - regard. Elle-même précise qu'il s'agit de la faculté de son âme : wzrok mej duszy-le regard de mon âme $(57,80$, $231,278,334,1239,1632,1327)$. Sur le regard de SF, il n'y a pas de prédications qui expriment une propriété, sauf wzrok wierności - regard fidèle (1239).

b) Les prédications métonymiques à la voix passive ou à la voix active

Les prédications à la voix passive réalisant une métonymie discursive sont constatées pour les verbes spuścić wzrok na-baisser le regard sur, zwrócić wzrok na - tourner le regard vers. Dans le premier cas, ils forment des syntagmes : mieć wzrok spuszczony na - avoir le regard baissé sur (408, 1284). Dans le deuxième cas, ces verbes entrent dans des constructions passives polonaises qui soit possèdent des constructions françaises parallèles (wzrok Pana jest zwrócony na - le regard du Seigneur est tourné vers (90)), soit sont traduites par des 
constructions à la voix active (lagodne spojrzenie Jezusa zwrócone jest $w$ dusze [Faustyny] - Jésus adresse le doux regard à l'âme [de Scur Faustine] (383)). La voix passive est aussi réalisée par le verbe pronominal réfléchi en polonais qui a un autre équivalent français à la voix passive, comme par exemple : Takie spojrzenie surowego Sędziego nie tyczylo się [siostry Faustyny] - Le regard d'un Juge sévère n'était pas dirigé vers [Sceur Faustine] (383).

La liberté discursive de la prédication métonymique réalisée par la voix passive est illustrée par le verbe spoczywać dans wzrok / spojrzenie Jezusa spoczywa na à la voix active qui est traduit en français selon les trois constructions possibles en fonction du contexte : la voix passive dans mon regard est posé sur lui jour et nuit (86), le pronom passif dans mon regard se pose sur cette maison avec prédilection (280) et la voix active dans Mon regard repose sur toi avec bienveillance (1700).

c) Deux prédications différentes en fonction de wzrok et de spojrzenie en polonais

Pour le prédicat polonais przenikać qui a pour sujet wzrok Boga - regard de Dieu, il y a deux équivalents français : transpercer et pénétrer. L'action du regard de Dieu vise l'âme de la SF. Pour le premier : przenikać duszę na wskroś - transpercer l'âme $(101,324,852)$. Le deuxième équivalent français : przenikać duszę do najtajniejszych głębin - pénétrer jusque dans les profondeurs les plus secrètes de l'âme $(560,432)$.

L'activité du regard de Jésus [spojrzenie Jezusa] est aussi celle d'apporter à l'âme de SF des biens spirituels (joie, amour). Elle est exprimée par le prédicat polonais napetniać qui a deux équivalents français : combler $(334,560)$ et remplir (758). Un autre prédicat polonais exprime la même activité du regard et des paroles de Jésus dans (858) : wprowadzić w duszę (sitę i moc, odwagę i dziwna ufność, $\dot{z} e$ ) - donner à l'âme (de la force et de la puissance, du courage et une étrange confiance que).

d) Les nomina actionis introduits par les verbes de perception visuelle ou de sentiments dans les constructions complétives révèlent les particularités syntaxiques qui mettent en relief le statut de nominalisation en polonais

Dans l'exemple Wewnętrznie widziałam spojrzenie Boga na nas z wielkim upodobaniem - J'ai vu intérieurement que Dieu nous regardait avec une grande prédilection (1439), le complément du verbe polonais widzieć est traduit par la phrase complétive canonique en français introduite par le verbe. Voir aussi l'exemple (1025).

Les parallélismes syntaxiques sont observés pour les verbes de sentiments $c z u c ́$ - sentir où la phrase complétive en français est soumise à la transformation infinitive : ... czulam spojrzenie Pana w głab serca mego - ... j'ai senti le regard 
du Seigneur scruter le fond de mon coeur (346). Le traducteur français restitue le verbe exprimant l'activité du regard - scruter (1391), mais aussi pénétrer (432, 758) - conformément aux prédications analysées en c).

Pour conclure les prédications métonymiques, nous constatons que celles-ci concernent les nomina actionis au sens 'action de V' qu'elles mettent en relief par le procédé de métonymie discursive caractérisée par une liberté de choix des constructions syntaxiques des verbes collocatifs et des nominalisations des prédications élémentaires de la perception visuelle.

\subsection{WIDZENIE / VISION : PRÉDICATION MÉTONYMIQUE SUR LE RÉSULTAT DE L'ACTION PERCEPTIVE}

Les mots widzenie / vision sont polysémiques dans les deux langues. Le sens dérivationnel du mot widzenie est défini en polonais par les gloses 'to, co widzimy, choć nie istnieje w rzeczywistości' (Slownik Języka Polskiego PWN, désormais $S J P P W N$ ) ou 'coś, co ktoś widzi, np. we śnie, w stanie halucynacji lub objawienia, a czego nie mogą zobaczyć inni' (Wielki Stownik Języka Polskiego, desormais $W S J P$ ). Les rédacteurs des deux définitions indiquent l'objet de l'action de voir et soulignent qu'il n'existe pas dans la réalité (SJP $P W N)$ ou qu'on voit cet objet dans un état d'hallucination ou de révélation et que les autres ne peuvent pas le voir $(W S J P)$. Le mot synonyme wizja, emprunté au latin, est défini aussi comme une représentation des événements qui doivent se produire dans l'avenir (SJP PWN) ou comme une image de quelque chose que quelqu'un voit bien que cela n'existe pas dans la « réalité objective » (WSJP). Dans le dictionnaire français, nous trouvons l'étymologie du mot vision qui est emprunté au latin visio 'action de voir, vue', 'ce qui se présente à la vue, image, simulacre des choses'. Les rédacteurs du $T L F i$ donnent aussi le sens spécialisé 'fait de voir Dieu', 'représentation d'ordre surnaturel apparaissant aux yeux ou à l'esprit'.

C'est bien dans ce sens spécialisé - qui n'a pas été noté dans les dictionnaires polonais - qu'est employé par SF le mot widzenie dont elle parle elle-même, donnant aussi le contenu de ce qu'elle voit. Sœur Faustine décrit le contexte dans lequel elle a ces visions : comme si son esprit « était transporté dans un autre monde » (30), il s'unit à Dieu $(472,882)$. Elle définit ces visions, qui sont purement spirituelles (835) ou « intérieures » (883), comme « connaissance » (742) : « c'est une illumination soudaine que Dieu m'accorde à cet instant» (835). Elle explique aussi : «bien que je ne les perçoive pas avec les sens, toutes les choses sont pour moi plus réelles et plus claires que si je les voyais avec les yeux » (882). 
Les verbes collocatifs avec ces noms expriment les prédications sur les actions de Dieu et sur les expériences de Sœur Faustine d'une part et, d'autre part, sur la vision elle-même.

a) Pour les prédications sur Dieu : Bóg udzielać SF widzenia-Dieu prodiguer à SF les visions $(130,835)$. Pour les prédications sur SF, c'est d'abord le verbe mieć - avoir (exprimant la conséquence de l'action de Dieu ci-dessus) : [dusza] wiele ma widzeń zmystowych i umystowych... - [l'âme] a beaucoup de visions sensibles et spirituelles (121), (cf. 125). D'autres verbes expriment ses activités localisées dans le contenu de la vision :

- l'activité perceptive : widzieć kogoś / coś w widzeniu - voir quelqu'un / quelque chose dans la vision $(258,61,573)$;

- l'activité cognitive : znać kogoś / coś w widzeniu - connaître quelqu'un I quelque chose dans la vision (263), par une vision (563); znać kogoś / coś przez widzenie - connaître quelqu'un / quelque chose grâce à une vision (53), par une vision (61).

b) Les prédications sur la vision se réalisent avec différents prédicats :

- le verbe copule być - être introduisant l'adjectif désignant une propriété pertinente : widzenie moje jest czysto duchowe - ma vision est purement spirituelle (835); widzenia moje sq czysto wewnętrzne - mes visions sont purement intérieures (883);

- les prédicats (napetnić - remplir, zalać - inonder) désignant une activité de la vision qui est celle d'apporter à l'âme un bien : widzenie to napetnito duszę moja radościa ... - cette vision a rempli mon âme de joie... (88) ; to widzenie [...] zalało duszę moja szczęściem niepojętym... - cette vision [...] a inondé mon âme d'un bonheur inimaginable... (472);

- le prédicat de répétition : powtórzyć się - se répéter (347);

- le prédicat zniknać - disparaître au temps passé accompli : widzenie zniklo - la vision a disparu $(31,330,348,447,616,846)$.

Sœur Faustine emploie encore les mots wizja, zjawisko et objawienie, mais ils signalent un tout autre contexte que celui de l'image donnée dans les rencontres avec Jésus. En français, ils ne sont pas spécifiés par d'autres lexèmes : wizja désigne quelque chose qui se déroule indépendamment de la rencontre (135), zjawisko désigne une scène avec d'autres personnages (416), objawienie est employé par quelqu'un d'autre (1422). Sœur Faustine réserve donc le nom d'action widzenie au contenu de ce qu'elle voit pendant les rencontres avec Jésus et démarque les autres contenus avec d'autres nomina actionis, ce qui n'est pas marqué par les noms français : tous sont traduits par vision, pourtant pour le mot objawienie il existe un autre équivalent - révélation. 


\section{Conclusion}

Au terme de la description détaillée des nomina actionis des verbes de perception visuelle relevés dans un texte bilingue parallèle, nous pouvons formuler deux types de conclusions. La première concerne la sémantique de ces noms, propre à chaque langue : il faut distinguer d'une part le sens lexical donné par la base verbale avec les participants nécessaires et, d'autre part, les changements de sens de ces nomina actionis résultant de la métonymie située dans la dimension ontologique ('sens' - 'action'), actancielle ('action' - 'résultat d'action') et discursive (la mise en relief de l'action). La deuxième conclusion formulée dans une dimension plus large porte sur le caractère par excellence communicatif des noms tels que spojrzenie - regard. Il n'est pas possible de s'arrêter au niveau de l'analyse lexicale et syntaxique de ces noms. Nous avons vu que leur signification complète se réalise dans l'échange entre les personnes qui communiquent par le regard un savoir, conformément à l'ontologie des yeux et à la perception de la réalité qui dépasse l'activité sensorielle de l'organe de la vision.

\section{Références bibliographiques}

\section{Ouvrages et articles}

HONOWSKA, Maria (1979), Ewolucja metod polskiego stowotwórstwa synchronicznego w dziesięcioleciu 1967-1977, Wrocław, Zakład Narodowy im. Ossolińskich.

HUYGHE, Richard (2014), "La sémantique des noms d'action : quelques repères ", Cahiers de lexicologie, $\mathrm{n}^{\circ} 105$, pp. 181-201.

ŚLIWA, Dorota (2000), Aspects dénominatifs de la morphologie dérivationnelle (étude des noms d'artefacts en français et en polonais), Lublin, RW, KUL.

ŚLIWA, Dorota (2002), «Un point de vue sur les dénominations des sentiments et des attitudes en polonais et en français », in Points communs : linguistique, traductologie, glottodidactique (A. Kacprzak éd.), Łódź, Wydawnictwo Biblioteka, pp. 275-284.

WASZAKOWA, Krystyna (2015), « Pragmatyka słowotwórczego działania (Kontynuacja myśli Marii Honowskiej o technikach stawania się form słowotwórczych) », LingVaria, $\mathrm{n}^{\circ}$ 19, pp. 99-108.

\section{Dictionnaires}

SJP PWN : Stownik Języka Polskiego, www.sjp.pwn.pl. WSJP : Wielki Słownik Języka Polskiego, www.wsjp.pl.

TLFi : Trésor de la Langue Française informatisé, www.atilf.atilf.fr. 


\section{Sitographie}

www.dictionnaire.academie-medecine.fr/?q=oeil.

www.dictionnaire.doctissimo.fr/definition-oeil.htm.

www.dictionnaire.doctissimo.fr/definition-vision.htm.

www.faustyna.pl/zmbm/dzienniczek-sw-siostry-faustyny.

www.faustyna.pl/zmbm/fr/texte-integral-du-petit-journal. 